\title{
Research on the Construction of Security Constrained Economic Dispatch Scenarios for Large-scale Power Grid
}

\author{
Guodong HUANG ${ }^{1}$, Dan XU ${ }^{1}$, Zhiqiang $\mathrm{LUO}^{2}$, Qiang DING ${ }^{1}$, Min $^{2}{ }^{3}$, and Hongtu ZHANG ${ }^{3}$ \\ ${ }^{1}$ Power Automation Department, China Electric Power Research Institute, Beijing, China \\ ${ }^{2}$ Power Dispatch and Control Center, the State Grid Corporation of China, Beijing, China \\ ${ }^{3}$ Power Dispatch and Control Center, the State Grid SICHUAN Electric Power Company, State Grid Corporation of China, Chengdu, \\ China
}

\begin{abstract}
Large-scale power grid security constrained economic dispatch is an effective technical means to solve the optimal allocation of resources in a large area. Based on the characteristics of dispatching plan in the State Grid Corporation, a processing strategy for optimizing objectives, planning adjustments, and basic data integration is proposed. A method of constructing a large-scale power grid security constrained economic dispatching scenario based on strategies combination is established, and the adaptability of different scenarios and the difficulty in constructing the corresponding model are analyzed. Multi-scenario construction aims at solving different problems in different scenarios and is a useful method for improving the availability and practicality of large-scale power grid security constrained economic dispatching technologies.
\end{abstract}

\section{Introduction}

With the rapid development of China's power industry, on the power supply side, large thermal power plants, large hydropower stations, and large wind farms have been gradually completed. The demand of transprovincial and trans-regional energy consumption has further increased. On the grid side, The construction of UHV interconnected power grids continues to deepen, and the scale of power grids develops rapidly. The structure of power grids has become increasingly complex and the nationwide networking has basically taken shape. The mutual influence and interaction of the power grids at all levels are further enhanced, and the characteristics of the power grid shift from the region mode to the overall mode.

The dispatching plan is an important task concerning the safe and economical operation of the power system and is one of the core businesses of the power control center in China. The supporting technologies in this field include the commonly used SCED (Secure Constrained Economic Dispatch) technology that uses the provincial area as the balance unit. However, this technology has shown some limitations in the trans-provincial and transregional energy consumption and multi-level integrated operation of the power grid. One way to solve the above problem is to change the scope of use of the SCED technology and extend it to to a larger grid. However, the large-scale SCED problems will bring great difficulties to data collection, model construction and solution [1-2]. In addition, the objective factors such as the multi-level organizational model of the dispatching center and differences in the balance strategy of the dispatch region will bring more difficulties to practical application.

At present, most of the related researches are started from decomposition and coordination. In [1], a method of decomposition and coordination for large grids based on price response functions is proposed. It decomposes the security constrained economic dispatch model for superlarge grids into the main problems of the upper-level tielines and the provincial-level unit planning sub-problems. Examples show that this method can effectively improve the economic dispatching efficiency of large-scale power grids, but this method does not consider China's power grids. The actual operation is not strong. Literature [2] proposed a large-scale decentralized unit combination model. In Europe and the United States, due to the continuous expansion of the electricity market, the main problems it faces are the joint optimization between different international and regional market rules, and the focus is on the solution method of the decentralized model. In [3], from the point of view of overall grid coordination, the development of tie line planning seeks to solve the problem of optimal allocation of global resources. However, the simulation example still stays between the two interconnected provinces, and it is difficult to adapt to the large-scale grid problem such as " East China- South China- Central China" interconnection. The literature [4-7] focuses on the study of the solution strategy for large-scale unit combination problems, but does not consider all practical constraints and grid control modes. The literature [8-11] focuses on the study of the absorption of uncontrollable renewable energy sources from models and algorithms. On the whole, the 
current economic constraints of security constraints on large-scale power grids have not been properly resolved.

According to China's power grid dispatch organization mode, it is currently divided into nationalregional and provincial dispatches. National-regional dispatch is mainly responsible for compiling the regional and inter-provincial tie-line plans, which embodies the inter-provincial electricity trading or trans-provincial and trans-regional consumption of large power sources. Provincial dispatch then compile their own unit plans based on load forecasting and exchange plans. According to the actual situation, if we want to carry out the entire grid security constrained economic dispatch, at least we need to consider the following additional issues: (1) optimization target design; ( 2 ) large-scale power system balance strategy design; (3) inter-provincial and large-area tie-line plan adjustment principles; (4) processing methods for power supply of different schedule relationship; ( 5 ) large-scale power grid network simplified methods applied to the planning requirements. With the deployment of smart grid dispatching and control systems within the State Grid Corporation of China, the basic data collection of dispatching plans in the provinces has been basically solved. This paper mainly proposes solutions to other problems and constructs usage scenarios for large-scale power grid security constraints and economic dispatch in a demand-combined manner. The paper analyzes the data requirements of different scenarios, the problems and the ease of model construction for the "Three Chinas" actual grid model. Enhance the availability and practicability of large-scale power grid security constrained economic dispatch technology under different dispatching modes by means of multi-scenario construction.

\section{Strategic design}

\subsection{Optimize objective design}

Usually, the objective of SCED optimization is to purchase the best price for the entire grid and optimize the coal consumption. Taking into account China's actual scheduling strategy, the SCED optimization goals in the province are mostly to consider energy-saving scheduling model of open and impartial power dispatch. However, since SCED users in the entire grid are usually in the overall scope of the decision-making problem, in addition to the provincial "open and impartial power dispatch" optimization goals, according to actual research, SCED of the whole network should add two optimization goals, namely, the regeneration of clean energy consumption and the maximization of power flow margins of key transmission sections.

The optimize objective of open and impartial power dispatch :

$$
f_{1}=\sum_{t=1}^{N_{r}} \sum_{i=1}^{N_{G}}\left(B_{0, i}+\sum_{k=1}^{K} \int b_{i, k}\left(p_{i, t}\right)\right)
$$

Where $N_{G}$ is the number of generator units; $N_{T}$ is the period of the study cycle, $B_{0, i}$ is the power generation cost constant for the i-th unit, $b_{i, k}\left(p_{i, k}\right)$ is the power generation micro rate curve for the $k$-th section of the $i$-th unit, and similar power generation micro-increasing rates for the same installed capacity, $k$ is the segment number of the multi-stage fee growth rate curve, $p_{i, t}$ is the unit output.

The objective function of maximizing the consumption of renewable energy:

$$
f_{2}=\alpha \sum_{t=1}^{T}\left(P_{W, t}^{\prime}-P_{W, t}\right)
$$

Where $\alpha$ is the penalty coefficient, $P_{W, t}$ is the total amount of wind power actually called by the wind farm during $t$ period, $P_{W, t}^{\prime}$ is the predicted wind power of the wind farm during period $t$.

The objective function of maximizing power flow margins of key transmission sections:

$$
f_{3}=\beta \sum\left(L_{t}-L_{t, \lim i t}\right)
$$

Where $\beta$ is the penalty coefficient, $L_{t}$ is the flow of the section and $L_{t, \lim i t}$ is the section limit during period $t$.

Therefore, there are at least three kinds of optimization objectives that can be selected: (1)Consider the energy-saving of open and impartial power dispatch for thermal power. (2) Maximize reproducible clean energy consumption in the entire grid. (3) Maximize the power flow margin of key transmission sections.

\subsection{System balancing strategy}

In the traditional province-based SCED model, the balancing strategy is that the total output of the units in the area is equal to the total load demand in the area, as shown in the following formula.

$$
P_{\text {load }, c, t}=\sum_{i \in c} P_{i, t}-T_{c, t}
$$

In the formula, $P_{\text {load }, c, t}$ is the total load demand of provincial $c$ at time $t ; P_{i, t}$ is the planned output of unit $i$ in province $c$ at time $t ; T_{c, t}$ is the outgoing power of provincial $c$ at time $t$ (negative when received) power . Usually $T_{c, t}$ is the data dispatched by the upper-level dispatcher and is balanced according to the determined value.

The above $T_{c, t}$ is a determined value in the model, which is a key factor that can deal with the whole grid decomposition. When the global optimization calculation 
is performed in the entire grid, it is in fact the optimization of the outgoing (received) power $T_{c, t}$ for each province. A simpler processing method is still to use the balancing strategy of formula (4) to extend the province $c$ to the global grid scope that needs to be optimized. The total provincial load forecast is added as the balance, and the power transmission between the grid and the external grid still uses known values. At this point, when the optimization is completed, $T_{c, t}$ can be obtained through load flow calculation. This approach is simple and straightforward, but the value of $T_{c, t}$ may deviate far from the actual tie-line plan. The reason is that according to the traditional SCED optimization goal, when the power trading related constraints are not added, the tie-line power will be completely determined by the power structure of each province. This is inconsistent with the formation mechanism of China's tie-line plan, which will cause difficulties in its use.

One solution that can solve the above problem is to directly add the constraint of the cross section of the tieline, and make the tie-line plan within the acceptable range in the constraint form, as shown in the following formula.

$T_{c, t, \min } \leq T_{c, t} \leq T_{c, t, \max }$

Where $T_{c, t, \min }$ and $T_{c, t, \max }$ are the minimum and maximum powers that can be sent (received) at the time $t$ in the province $c$.

In another solution, the provinces retain the balance constraint and use the tie-line as the equivalent power source to preserve the global optimality. Equivalence means that the physical lines with both ends in the same province are merged into a pair of equivalent power supplies. At this time also meet the following constraints: (1) two-terminal coupling constraints, that is, under the premise of ignoring grid losses, the sum of the power transmitted at both ends of the line is 0; (2) power dispersion injection characteristics, in order to carry out the power flow calculation, the optimize power are distributed at the physical line access points according to the equivalent historical statistics. According to this idea, the SCED scenario of the whole grid needs to add positive-negative coupling equivalent unit information and the power distribution factor.

There are at least three possible system balancing strategies that can be adopted: (1) The unified balance of the whole grid obtained from the traditional provincial balance promotion. (2) Add the entire grid balance of the tie-line constraint. (3) Tie-line participates in system balance as an equalizer.

\subsection{Adjustment principle of tie-line plan}

In the balance strategy mentioned above, when the tieline is used as an equalizer to participate in the system balance, the tie-line power is classified as a demand variable, and the emphasis is on the adjustment zone of the given tie-line power. However, in fact, the tie-line plan is mainly based on the cross-regional and interprovincial balance of large-scale power supply plans and allocation strategies and power exchange between provinces [3]. This shows that the adjustment zone defined for tie-line must meet certain constraints.

According to the actual situation, the power composition of the tie-line can be divided into two parts: provincial power trading and cross-provincial crossregional balanced power supply, as shown in the following formula.

$T_{l, t}=T_{l, s, t}+T_{l, u, t}$

$T_{l, t}$ is the tie-line power; $T_{l, s, t}$ is the power trading decision quantity; $T_{l, u, t}$ is the cross-provincial crossregional power decision quantity. If the transaction is adjustable, an adjustable interval of $T_{l, s, t}$ can be given based on the rigidity of the transaction. If the large-scale cross-provincial power supply in the entire grid plans to do a fixed output processing (in fact, most of the largescale cross-provincial power supplies are large-scale thermal power and hydropower groups, and usually plan values are prepared in advance), then $T_{l, u, t}$ does not have adjustment zone. If this part of the power supply is also involved in the optimization, the adjustment interval should be associated with the amount of change in the value, as shown in the following equation.

$$
\Delta T_{l, u, t}=\sum \alpha_{i, l, t} \cdot\left(P_{i}-p_{i, i n i}\right)
$$

$\Delta T_{l, u, t}$ is the adjustment zone of cross-provincial cross-regional power supply decision volume, $P_{i}$ is plan of the cross-provincial cross-regional power supply $i$, $p_{i, \text { ini }}$ is initial plan of the power supply $i, \alpha_{i, l, t}$ is the power distribution coefficient assigned to the line $l$ for the unit $i$ at time $t$.

In practice, the tie-line is also divided into largeregion tie-line and inter-provincial tie-line. There is also an associated relationship between them. Usually, the power of the line transmission in the region is mostly consumed by multiple provinces within the region. Therefore, the adjustment of the power of the tie-line in the region will inevitably lead to the adjustment of the power of the inter-provincial tie-line in the region. In practice, the power of the regional tie-lines will be apportioned among the intra-provincial tie-lines within the region, so the adjustment relationship is as follows.

$$
\Delta T_{a, t}=\sum \gamma_{a, l, t} \cdot\left(T_{l, t}-T_{l, t, i n i}\right)
$$

$\Delta T_{a, t}$ is the adjustment amount of the region tie-line $a$; $T_{l, t}$ is the plan of the tie-line $l$ in the province at time $t$ $T_{l, t, i n i}$ is the initial value of the tie-line $l$ in the province 
at time $t ; \gamma_{a, l, t}$ is the apportionment factor of tie-line $l$ in the province at time $t$.

The adjustment method of the tie-line plan is determined by three factors. This paper selects three adjustment models: (1)The power trade can be adjusted, and cross-provincial large-scale power supply plans cannot be adjusted. (2)Both are adjustable. (3) Both are adjustable and take into consideration the relevance of the tie-line between the major regions and the provinces.

\section{Basic data integration}

At present, the basic data of the whole grid dispatch plan comes from the provincial data reporting, and has now been applied to the quantitative security check of the “ East China- South China- Central China”, and the verified accuracy of planned flow has been recognized [12]. This has ensured the feasibility of basic data collection to a certain extent. However, due to the substantial differences between SCED and security check, it is not possible to directly copy the way of handling the security check. It is also necessary to consider the solution that is appropriate to the scheduling plan.

\subsection{Unit data process}

Through the information reported by the provinces, the basic data of the unit is obtained, and the unit data must be specially processed, because the unit has the following special attributes in the dispatching plan: in terms of the scheduling relationship, the unit has local balance unit and cross-provinces balance unit, and different scheduling attributes will determine the way in which it participates in system balancing; in terms of network model construction, the unit has modeling units and unmodeled units, which will determine its impact on network flows and model accuracy. Therefore, in the SCED scenario construction of the entire network, differences need to be considered for the above scheduling attributes of the unit.

For the cross-provincial cross-regional balanced unit, the method of binding with each related tie line is used, as shown in the following formula.

$$
T_{l, i, t}=\alpha_{i, l, t} \cdot P_{i}
$$

In the formula, $T_{l, i, t}$ represents the power value of the tie- line $l$ determined by the unit $i$ at time $t$; $P_{i}$ is the planned output of the unit $i ; \alpha_{i, l, t}$ is the power distribution coefficient of the unit $i$ assigned to the tieline $l$ at time $t$. Another issue that needs special attention here is that when the unit power is reflected in the tie-line plan, it can no longer participate in the system balance in the unit form, otherwise it will cause repeat calculation of power supply output. However, it must participate in system power flow calculations, otherwise it will cause power imbalance in the power flow calculation.

For the unmodeled units, the power plant reported as a fixed output processing in the current provincial dispatch plan. In the whole-grid SCED, it also exists in the fixed output mode. Since it does not affect the optimization of the entire grid resources and the power flow analysis, the unmodeled units will adopt the intrastate packaging processing mode, as shown in the following formula.

$$
P_{s, c}=\sum_{i \in c} p_{i, s}
$$

In the formula, $p_{i, s}$ is output of the unmodeled unit $i$, this value has been obtained in advance; $P_{s, c}$ is the total output of unmodeled small units within the provincial $c$. At this point, the total output in the scene replaces the unmodeled small units.

From the analysis, we can see that the processing of the different scheduling relationship units will depend on the choice of balancing strategy: (1) Adopt the unified balance mode of the whole grid, you can not consider the characteristics of cross-provincial cross-regional balance, that is, the unit does not need to be converted into a tieline plan to directly participate in the balance. (2) Considering the constraint of inter-area and interprovincial tie-lines, and adjusting according to actual conditions, the different scheduling attributes of the unit must be considered.

\subsection{DC transmission system process}

In the interconnection of large-scale power grid, the interconnection between regions through a HVDC transmission system is a common approach. Because with the further development of high-power power electronic fully-controlled switching device technology and the research of new control strategies, DC transmission can not only achieve energy transmission, but also make it have certain schedulability characteristics, which is different from the AC connection line. Therefore, it is necessary to handle the HVDC transmission system separately.

The HVDC transmission system is equivalent at the AC converter valve. If the DC power is set as the SCED optimization variable of the whole network, the equivalent unit processing mode is still used. However, compared to the AC line, the DC line plan will be subject to a series of constraints such as DC operating characteristics, methods, and operating procedures. If it is to be included in the optimization, the DC operating characteristics, methods, and procedures must be modeled in detail, converted into unit output caps, and the characteristics of a series of scheduling plans such as the prohibition of operating areas and climbing characteristics should be incorporated into the model constraints. This will be a very complicated issue. We will only give special attention to this issue and will not discuss it in depth.

Therefore, DC should also have the following two processing methods: (1) If the DC system does not participate in the optimization decision, the processing method can be simplified, and processing can be performed according to the fixed output equivalent, but at 
this time, the optimization space between the areas is reduced. (2) DC takes part in the optimization as a variable, and it is necessary to consider various constraints and it is more complicated.

\subsection{Power network model process}

For large-scale power grid such as "East China- South China- Central China ", the consideration of the power network model in SCED will bring about enormous calculation [1]. However, if the network constraints are not considered, some of the value of SCED optimization will be lost.

Decomposing large-scale power network into provincial-scale power netwrok using equal-value mode in planning flows is the currently method adopted by single province SCED. If the balancing strategy of the second scenario in Section 1 of this paper is followed, the network can be decomposed into provinces. This will greatly reduce the network analysis calculation time.

On the other hand, since the users of the entire network SCED are mostly dispatch planners, according to the focus of the users, the key lines can be selected to analyze in planning flow. These key lines are mainly distributed in the large power network near area and the near area of the UHV AC/DC, so the lines that need attention are in fact relatively fixed.

First select the specific line according to the principle. Principle One: Pay attention to heavy load lines. The operation of the power grid has a certain degree of continuity. When the mode of operation does not change much, the heavy load condition of the line flow can be determined based on the historical trend and included in the concerned lines. In principle two, when there are major changes in the operation of the power grid, planners can often determine in advance the changes in the flow of critical lines brought about by the changes, so as to determine the lines that need attention. After selecting the specific line that needs attention, you can calculate the branch current according to DC sensitivity analysis, as shown in the following formula.

$$
P_{z, t}=\sum \beta_{i, z} \cdot P_{i}+P_{\text {load }, z, t}
$$

In the formula, $P_{z, t}$ is the power flow of line $z$ at time $t ; \beta_{i, z}$ is the sensitivity of unit $i$ to line $z ; P_{l o a d, z, t}$ is the contribution of all loads to this line. Since $\beta_{i, z}$ and $P_{l o a d, z, t}$ are only related to the power network, which can be calculated before SCED optimization.

In dealing with the network model, there are at least the following types of processing: (1) Without considering network constraints, ie without planning power flow analysis, SCED problems are converted into ED problems; (2) Consider the whole network model; (3) Consider the province-wide model according to the whole network; (4) Consider the simplified model of the whole network divided by province.

\section{SCED scenario construction for large power grid}

Based on the above analysis, large-scale SCED need to face many strategies and basic data processing problems. Different strategies and processing methods will constitute different planning scenarios. Combined with the actual situation and the relevance, this paper has built several typical scenarios for different analysis purpose. The combination method is shown in Table 1.

Table 1. List of scenarios.

\begin{tabular}{|c|c|c|c|c|c|}
\hline \multirow{2}{*}{$\begin{array}{c}\text { Scenario } \\
\text { s }\end{array}$} & $\begin{array}{c}\text { Main Considerations } \\
\text { Balance } \\
\text { strategy }\end{array}$ & $\begin{array}{c}\text { Tie-line } \\
\text { adjustmen } \\
\boldsymbol{t}\end{array}$ & $\begin{array}{c}\text { Unit } \\
\text { schedulin } \\
\boldsymbol{g} \\
\text { relationsh } \\
\text { ip }\end{array}$ & DC & $\begin{array}{c}\text { Power } \\
\text { networ } \\
\boldsymbol{k}\end{array}$ \\
\hline 1 & $(1)$ & $(1)$ & $(1)$ & $(1)$ & $(2)$ \\
\hline 2 & $(2)$ & $(2)$ & $(1)$ & $(1)$ & $(2)$ \\
\hline 3 & $(3)$ & $(2)$ & $(2)$ & $(1)$ & $(2)$ \\
\hline 4 & $(3)$ & $(2)$ & $(2)$ & $(2)$ & $(2)$ \\
\hline 5 & $(3)$ & $(3)$ & $(2)$ & $(2)$ & $(3)$ \\
\hline
\end{tabular}

Through analysis, the following conclusions can be drawn:

Scenario 1 directly extends the traditional provincial SCED problem to the whole network, and is suitable for analyzing the theoretical optimization results obtained in the whole network in accordance with the unit characteristics.

Scenario 2 implements the whole network SCED on the premise of satisfying the power trade adjustment. Because DC is not adjustable, the scenario can be defined as the optimal configuration of resources in the region under the precondition of adjusting the power trade.

Scenario 3 is the optimization of resources in the region under the premise of allowing both power trade and large power optimization adjustment.

Scenario 4 is based on scenario 3, since HVDC transmission is adjustable,it is a resource-wide optimization configuration within the whole network.

Scenario 5 is a resource-optimized configuration within the entire network that best fits the actual situation of the current dispatch in China.

The above lists the various scenarios that this article considers worth studying. It is not a collection of all scenes. Researchers can make different combinations of scenarios based on research needs. Because different scenarios have large differences in the constraints considered by the planning model, building a scenario according to requirements has certain practical significance.

\section{Acknowledgment}

Thanks for support from the science and technology program of State Grid Corporation of China (Research on Parallel Coordination Strategy and Key Techniques in Generation Scheduling over Large-scale Power Grids). 


\section{References}

1. LAI Xiao-wen, ZHONG Hai-wang, YANG Jun-feng et al. Decomposed Optimization Method over Largescale Power System Based on Price Response Function. Automation of Electric Power Systems, 37 (2013)

2. Mohammad Javad Feizollahi, Large-Scale Decentralized Unit Commitment. August 27, 2014

3. Xu Dan, LI Xiao-lei, DING Qiang Optimization of Tie Line Hierarchical Schedule Based on Networkwide Coordination. Automation of Electric Power Systems, 38 (2014)

4. Wang B, Ye X, Xia Q, et al. Security-Constrained Economic Dispatch With AC/DC Interconnection System Based on Benders Decomposition Method. Proceedings of the Csee, 36 (2016)

5. Ding Q, Li K, Zhu Z, et al. Economic Dispatch Optimization Method for Large-scale Power Grid Based on Variable Dimension Reduction. Automation of Electric Power Systems, 41 (2017)

6. Kargarian A, Fu Y, Li Z. Distributed SecurityConstrained Unit Commitment for Large-Scale Power Systems. IEEE Transactions on Power Systems, 30 (2015)

7. Zhao W, Liu M. Cutting Plane Consensus Algorithm for Decentralized Dynamic Economic Dispatch in
Multi-area Power systems. Proceedings of the Csee, 36 (2016)

8. Ahmadi-Khatir A, Conejo A J, Cherkaoui R. MultiArea Unit Scheduling and Reserve Allocation Under Wind Power Uncertainty. IEEE Transactions on Power Systems, 29 (2014)

9. Fu Y, Liu M, Li L. Multiobjective Stochastic Economic Dispatch With Variable Wind Generation Using Scenario-Based Decomposition and Asynchronous Block Iteration. IEEE Transactions on Sustainable Energy, 7 (2015)

10. Li Z, Shahidehpour M, Wu W, et al. Decentralized Multiarea Robust Generation Unit and Tie-Line Scheduling Under Wind Power Uncertainty. IEEE Transactions on Sustainable Energy, 6 (2015)

11. Saurabh Tewari, Charles J. Geyer, Ned Mohan ,A Statistical Model for Wind Power Forecast Error and its Application to the Estimation of Penalties in Liberalized Markets, IEEE TRANSACTIONS ON POWER SYSTEMS, 26 (2011)

12. Men D Y, Ji X, Cai Z, et al. Expert Library-Based Data Verification for National-Regional-Provincial Power Grid Integration Security Correction. Advanced Materials Research, 805-806 (2013) 